\title{
Associations between Caregiver-Perceived Delirium in Patients with Cancer and Generalized Anxiety in their Caregivers
}

\section{Citation}

Buss, Mary K., Lauren C. Vanderwerker, Sharon K. Inouye, Baohui Zhang, Susan D. Block, and Holly G. Prigerson. 2007. "Associations Between Caregiver-Perceived Delirium in Patients with Cancer and Generalized Anxiety in Their Caregivers." Journal of Palliative Medicine 10, no. 5: 1083-1092.

\section{Published Version}

doi:10.1089/jpm.2006.0253

\section{Permanent link}

http://nrs.harvard.edu/urn-3:HUL.InstRepos:13956244

\section{Terms of Use}

This article was downloaded from Harvard University's DASH repository, and is made available under the terms and conditions applicable to Other Posted Material, as set forth at http:// nrs.harvard.edu/urn-3:HUL.InstRepos:dash.current.terms-of-use\#LAA

\section{Share Your Story}

The Harvard community has made this article openly available.

Please share how this access benefits you. Submit a story.

Accessibility 


\title{
Associations between Caregiver-Perceived Delirium in Patients with Cancer and Generalized Anxiety in their Caregivers
}

\author{
MARY K. BUSS, M.D., M.P.H., ${ }^{1}$ LAUREN C. VANDERWERKER, Ph.D., ${ }^{1}$ \\ SHARON K. INOUYE, M.D., M.P.H., ${ }^{2}$ BAOHUI ZHANG, M.S., ${ }^{1}$ \\ SUSAN D. BLOCK, M.D., ${ }^{1}$ and HOLLY G. PRIGERSON, Ph.D. ${ }^{1}$
}

\begin{abstract}
Background: Delirium, a common complication of advanced cancer, may put caregivers at risk for poor mental health outcomes. We looked for a relationship between caregiver-perceived delirium in a patient with advanced cancer and rates of caregiver psychiatric disorders.

Methods: Using cross-sectional data from 200 caregivers of patients with cancer with a life expectancy of less than 6 months, we determined the frequency of caregiver-perceived delirium, which was defined as caregivers who reported witnessing the patient "confused, delirious" on the Stressful Caregiving Response to Experiences of Dying (SCARED) weekly or more often. We tested for associations between caregiver-reported delirium and presence of caregiver mental disorders, using the Structured Clinical Interview for the DSM-IV to diagnose mental disorders and caregiver burden, as measured by the caregiver burden scale (CBS).

Results: Of the 200 caregivers who completed the SCARED, $38(19.0 \%)$ reported seeing the patient "confused, delirious" at least once per week in the month prior to study enrollment and 7 (3.5\%) met criteria for generalized anxiety (GA). Caregivers of patients with caregiver-perceived delirium were 12 times more likely to have GA (odds ratio $[\mathrm{OR}]=12.12 ; p<0.01$ ). The relationship between caregiver-perceived delirium and caregiver GA persisted after adjusting for caregiver burden and exposure to other stressful patient experiences $(\mathrm{OR}=9.99 ; p=0.04)$.

Conclusions: This is the first report of an association between caregiver-perceived delirium and a caregiver mental health outcome. Further studies, using improved measures of delirium, are needed.
\end{abstract}

\section{INTRODUCTION}

D ELIRIUM is a common complication of cancer, occurring in $28 \%-50 \%$ of hospitalized patients with cancer $^{1-4}$ and up to $88 \%$ of patients with cancer prior to their death. ${ }^{5}$ In patients with advanced cancer, delirium is associated with decreased survival. ${ }^{6,7}$ Patients with delirium, based on their increased likelihood of being discharged to a facility rather than home, ${ }^{8}$ are less likely to be able to care for themselves. Because delirium can impair patient capacity, caregivers may take a more active role in medical decision-making for patients with delirium. Thus, delirium can increase the physical as well as the emotional and psychological burden of cancer caregivers, yet little is known about the association between delirium in patients and caregivers' mental health.

\footnotetext{
${ }^{1}$ Center for Psycho-oncology and Palliative Care Research, Division of Psychosocial Oncology and Palliative Care, DanaFarber Cancer Institute, Brigham and Women's Hospital, Harvard Medical School, Boston, Massachusetts.

${ }^{2}$ Department of Medicine, Beth Israel Deaconess Medical Center, Harvard Medical School, Aging Brain Center, Hebrew Senior Life, Boston, Massachusetts.
} 
The highly demanding nature of caregiving for dementia patients is widely recognized ${ }^{9-12}$ and may help inform work exploring the relationship between patient delirium and caregiver well-being. In one study of dementia caregivers, $43 \%$ of caregivers met criteria for depression just prior to a patient's death. ${ }^{13} \mathrm{Be}-$ havioral disturbances, such hallucinations and delusions of theft, have a strong association with poor caregiver outcomes in patients with dementia. ${ }^{14-16}$ Hospitalization of dementia patients and deterioration of the patient-caregiver relationship have also been shown to increase caregiver burden. ${ }^{17-19}$ Delirium often occurs in a hospital setting and is commonly characterized by hallucinations or paranoid delusions. Because many of the features of dementia that put caregivers at risk can also occur in patients with delirium, delirium seems likely to put caregivers at risk for distress. The fluctuating nature of delirium and the uncertainty of recovery-features not shared by dementia-may actually heighten ill effects on caregivers.

Only two research studies directly evaluate the effect of patient delirium on caregivers. ${ }^{20,21}$ Breitbart $^{20}$ documents moderate to severe levels of distress in $76 \%$ of caregivers shortly after a patient recovered from delirium, using a single item to measure caregiver distress. A second Japanese study, using a mailed questionnaire, found that over two thirds of bereaved family members experienced the presence of any delirium-related symptoms (except somnolence) as distressing or very distressing. Several features of this study call into question the generalizability of the results to caregivers of patients with cancer in the United States: (1) it was conducted using a Japanese sample; (2) all patients were cared for at specialized palliative care units and (3) recall bias, inherent in survey research, especially among the bereaved, could affect findings.

Given the prevalence of delirium in the advanced cancer population and the common features between dementia and delirium, further evaluation of the effects of delirium on cancer caregivers is warranted. In this study, using a secondary data analysis of a large study of patients with cancer and their caregivers, we test for a relationship between caregiver's perception of delirium in patients and rates of caregiver psychiatric disorders, using the Structured Clinical Interview for DSM-IV (SCID).

\section{METHODS}

\section{Study design and sample}

Data for this report came from the "Coping with Cancer Study" (MH63892, CA106370), an ongoing multisite, longitudinal investigation of the prevalence and treatment of mental disorders, mental health service use, quality of death, and bereavement adjustment among patients with advanced cancer and the individual they designated as their primary informal caregiver (e.g., spouse, adult child). The current report is focused on the baseline assessment of caregivers from three of the participating sites, namely Yale Cancer Center (New Haven, CT), the Veterans Affairs Connecticut Healthcare System Comprehensive Cancer Clinics (West Haven, CT) and the Parkland Hospital Palliative Care Service (Dallas, TX).

Caregivers were eligible if patients identified them as providing the majority of their unpaid, informal care. To be eligible, patients had to have advanced cancer as indicated by the presence of distant metastases and the failure of first-line therapy. Excluded were caregiver-patient dyads in which either individual was: (1) not proficient in English or Spanish, (2) met criteria for severe cognitive impairment at the time of study entry, using the Short Portable Mental Status Questionnaire, or (3) less than 20 years old. Based on the judgment of the interviewer, if a patient appeared to be too weak or incapacitated to complete the interview and/or provide reliable and valid responses, that patient was not approached. All study documents were approved by the Human Investigations Committee of Yale University and the participating institutions. At the time of study enrollment, caregivers reported on the following: (1) the presence of any mental health problem prior to the patient's cancer diagnosis, (2) the number and percentage of caregivers who discussed these problems with a mental health professional, (3) stressful patient experiences they observed in the previous month (the month prior to the study enrollment), including "delirium, confusion" and (4) the Structured Clinical Interview for DSM-IV (SCID) Axis I modules as a means of determining if caregivers met criteria for a psychiatric disorder at the time of study enrollment, by reflecting on their symptoms over the past month. Further details on the study recruitment and enrollment and the sample have been previously published. ${ }^{22}$

\section{Measures}

Demographics and patient-related variables. Information was collected on both the patients' and the caregivers' age, gender, race, level of education, and income. Caregivers were asked how many months they had been providing care for the patient, and whether or not the patient had health insurance. Information about the patients' cancer type was obtained from medical records. At the time of study enrollment, clinicians 
provided a rating of the patient's functional, using the Zubrod Performance Scale, which ranges from 0 (normal activity; asymptomatic) to 4 (100\% bedridden) and the Charlson Index of Co-morbidity. ${ }^{23}$

Caregiver-perceived delirium. Caregivers completed the Stressful Caregiving Response to Experiences of Dying (SCARED). ${ }^{24}$ The SCARED was used to assess caregiver exposure to patient distress, and the fear and helplessness evoked by these experiences. One item of the SCARED asks caregivers to record how often they witnessed the patient "confused, delirious" in the previous month on a Likert Scale $(0$, never; 1 , once or twice; 2 , every week; 3 , every day). For the purposes of this study, we considered patients in whom a caregiver reported the patient "confused, delirious" weekly or daily as having caregiver-perceived delirium.

Cognitive status measures. Because the SCARED measure described above provides a rather crude measure of delirium, we attempted to verify that the caregiver-perceived delirium captured only transient or fluctuating types of cognitive impairment, such as delirium and not more chronic forms of cognitive dysfunction, such as dementia. Several steps were taken in the study design to eliminate patients with dementia from participating. First, cognitive impairment was an exclusion criterion of the study. Before patientcaregiver dyads were approached, the research assistant (RA) contacted the patient's primary oncologist for permission to approach the patient and to ensure that patients met eligibility criteria. Oncologists were reminded, "Patients deemed too weak or cognitively impaired are excluded." Prior to approaching a patient or caregiver, the RA reviewed the medical record to confirm eligibility and patients with diagnosed dementia in their medical records were excluded.

When approaching the patients for enrollment, the RA began the process by having patients complete the Short Portable Mental Status Questionnaire (SPMSQ), a measure of cognitive function. ${ }^{25}$ Scores on the SPMSQ range from 0 to 10 ; a score of $8-10$ corresponded to intact cognitive function; $6-7$ with mild cognitive impairment; $3-5$ with moderate and $0-2$ with severe cognitive impairment. ${ }^{26,27}$ Patients with a score under 6 at the time of study enrollment were excluded from participation in the Coping with Cancer Study. The SPMSQ is not accurate as a screening tool for delirium because of the fluctuating nature of delirium; however, scores under 8 have been shown to correlate with higher rates of delirium. ${ }^{28}$

Patients with a SPMSQ score of 6 or above proceeded with the interview, which takes approximately
75 to 90 minutes to complete. The RA prompted patient responses. All the RAs have training and extensive experience in psychiatric interviewing techniques. RAs were aware of excessive time to respond, need for frequent prompting and other cues that indicated the patient responses may not be reliable. At the conclusion of the interview, RAs rated their confidence in the patient response on a 5-point Likert scale.

Major depressive disorder and anxiety disorder diagnoses. The Structured Clinical Interview for the DSM-IV (SCID) Axis I Modules ${ }^{29}$ was used to diagnose current psychiatric disorder among the caregivers. The SCID is a widely used instrument with proven reliability and validity $(\kappa=0.56$ for Generalized Anxiety Disorder; $\kappa=0.58$ for Panic Disorder ${ }^{29}$; $\kappa=0.92$ for Hamilton Rating Scale for Depression score of greater than 17 and SCID-diagnosed Major Depressive Disorder (MDD). The disorders assessed included MDD and the following anxiety disorders: posttraumatic stress disorder (PTSD), generalized anxiety disorder (GAD), and panic disorder (PD). Because the items on the SCID, as used in this study, only asked subjects to report on the presence of symptoms for the past month and a diagnosis of GAD requires at least 6 months of symptoms, we refer to subjects with a positive SCID for GAD as having GAD symptomatology or generalized anxiety, rather than the GAD itself.

Caregiver burden. The Caregiver Burden Scale (CBS), which has been validated for use in a variety of populations of caregivers, ${ }^{30,31}$ was used to measure the stress of caregiving. The 16 four-point Likert scale items of the CBS measure the physical, emotional, and instrumental (e.g., shopping, handling finances) tasks and their level of demand and difficulty for caregivers. ${ }^{31}$

\section{Statistical analyses}

The primary goal of this study was to test for associations between caregiver reports of delirium symptoms and caregiver psychiatric disorders. $\chi^{2}$, analysis of variance (ANOVA), or $t$ test statistics were used to determine whether caregivers who reported caregiverperceived delirium in the patient differed significantly from those who did not report caregiver-perceived delirium on the following demographics: gender, race, age, education, patient health insurance status, marital status, relationship to the patient, or recruitment site. We also analyzed a number of patient variables that have been shown in prior studies to correlate with delirium. Specifically, we tested for an association between caregiver-perceived delirium and patient age, 
gender, Zubrod performance status, stage of cancer and Charlson comorbidity index.

Bivariate logistic regression models estimated the relationship between caregiver-perceived delirium and the caregiver meeting criteria for any psychiatric disorder on the SCID. A separate logistic regression model estimated the bivariate association between caregiver-perceived delirium in the patient and caregiver burden. Prior to conducting the study, we planned to additional analyses for any psychiatric disorder that was significantly associated with reports of caregiver-perceived delirium, including associations with caregiver demographic characteristics and the psychiatric disorder. Additionally, for any psychiatric disorder that was significantly associated with care- giver-perceived delirium in bivariate analyses (i.e., generalized anxiety), we examined whether this association was mediated by the overall burden of caring for a seriously ill patient. A third logistic regression model included both caregiver-perceived delirium and caregiver burden to determine whether the presence of burden in the model would negate the significance between caregiver-perceived delirium and the outcome of interest. Finally, we conducted post hoc analyses looking at the relationship between generalized anxiety and reporting of other objective patient experiences to investigate whether the relationship with caregiver anxiety was unique to caregiver-perceived delirium or if caregivers with anxiety had also reported more exposure to other experiences assessed by the

Table 1. Sample Characteristics $(N=200)$ of Caregivers of Patients with Advanced Cancer

\begin{tabular}{|c|c|}
\hline Attribute & $\mathrm{n}(\%)$ \\
\hline Gender & $49(24.5 \%)$ \\
\hline Male & $49(24.5 \%)$ \\
\hline Female & $151(75.5 \%)$ \\
\hline \multicolumn{2}{|l|}{ Ethnicity } \\
\hline White & $127(63.5 \%)$ \\
\hline Black & $39(19.5 \%)$ \\
\hline Hispanic & $24(12.0 \%)$ \\
\hline Asian & $6(3.0 \%)$ \\
\hline Other & $4(2.0 \%)$ \\
\hline \multicolumn{2}{|l|}{ Relationship to patient } \\
\hline Spouse/partner & $114(57.0 \%)$ \\
\hline Child & $48(24.0 \%)$ \\
\hline Sibling & $13(6.5 \%)$ \\
\hline Other family member & $12(6.0 \%)$ \\
\hline Friend & $10(5.0 \%)$ \\
\hline Other & $3(1.5 \%)$ \\
\hline \multicolumn{2}{|l|}{ Age (years) } \\
\hline Mean (SD) & $49.1(13.5)$ \\
\hline Median (range) & $49.5(20-83)$ \\
\hline \multicolumn{2}{|l|}{ Education (years) } \\
\hline Mean (SD) & $13.0(3.4)$ \\
\hline Median (range) & $12(0-21)$ \\
\hline \multicolumn{2}{|l|}{ Income } \\
\hline$\$ 0-\$ 10,999$ & $24(12.0 \%)$ \\
\hline$\$ 11,000-\$ 20,999$ & $19(9.5 \%)$ \\
\hline$\$ 21,000-\$ 29,999$ & $14(7.0 \%)$ \\
\hline$\$ 30,000-\$ 50,999$ & $15(7.5 \%)$ \\
\hline$\$ 51,000-\$ 99,999$ & $47(23.5 \%)$ \\
\hline$\$ 100,000$ or more & $6(3.0 \%)$ \\
\hline Refused & $22(11.0 \%)$ \\
\hline Don't know & $36(18.0 \%)$ \\
\hline Missing & $17(8.5 \%)$ \\
\hline \multicolumn{2}{|l|}{ Treatment Center ${ }^{\mathrm{a}}$} \\
\hline Yale Cancer Center (CT) & $116(58.0 \%)$ \\
\hline VA CT Healthcare System Cancer Center (CT) & $13(6.5 \%)$ \\
\hline Simmons Comprehensive Cancer Center (TX) & $2(1.0 \%)$ \\
\hline Parkland Palliative Care Service (TX) & $69(34.5 \%)$ \\
\hline
\end{tabular}

${ }^{a}$ VA $=$ Veterans Health Administration, West Haven, CT. $\mathrm{SD}$, standard deviation. 
SCARED. We also tested for associations between caregiver anxiety and any patient variable (e.g., age, gender, performance status) that was associated with caregiver-perceived delirium. The final logistic regression model adjusted for all of the items on the SCARED, for caregiver burden and for any patient variable associated with caregiver anxiety.

\section{RESULTS}

A total of 200 caregivers had complete data for the variables of interest and were available for analyses. The characteristics of these caregivers are displayed in Table 1.

At the time of study enrollment, 35.9\% caregivers $(n=71)$ reported discussing a mental health problem with a health care professional prior to the patient's cancer diagnosis. Of these caregivers, 61 of them $(85.9 \%)$ discussed these problems with a mental health professional.

\section{Frequency of caregiver-perceived delirium}

Of the 200 caregivers who completed the SCARED, $38(19.0 \%)$ reported seeing the patient "confused, delirious" at least once per week in the month prior to study enrollment. To support the use of the SCARED item as a measure of delirium, we looked at the relationship between the SCARED measure of delirium and the SPMSQ score. Those with caregiver-perceived delirium were significantly more likely to score between 6 and 8 (indicative of mild cognitive dysfunction) on the SPMSQ than those without caregiver-reported delirium (15.6\% versus 0.7\%; Fisher's exact test $p<0.001)$. In only one case did an RA report little to no confidence in a completed patient interview (due to cognitive functioning), and this case was excluded from further analyses.

We analyzed a variety of demographic variables, in- cluding age, gender, race, educational level, health insurance status, marital status, and relationship to patient. None of these variables differed significantly in the group with caregiver-perceived delirium compared to those without caregiver-perceived delirium; therefore, we did not include the demographic variables in our regression models. In previous studies, certain patient characteristics, such as age, performance status, comorbidities, and survival have been shown to correlate with patient delirium. ${ }^{6,32} \mathrm{We}$ found no association between caregiver-reported delirium and patient gender $\left(\chi^{2}=0.07 ; p=0.79\right)$, patient age $(t=-0.16$; $p=0.87)$, or the Charlson co-morbidity index $(t=$ $-1.30 ; p=0.20)$. Caregiver-perceived delirium did correlate with higher (or worse) Zubrod scores $(r=$ $0.28 ; p<0.0001)$.

\section{Associations between caregiver-perceived delirium and caregiver mental health outcomes}

The relationship between psychiatric disorders in caregivers who reported caregiver-perceived delirium in the patient compared to caregivers who did not report caregiver-perceived delirium is shown in Table 2. Caregivers who reported caregiver-perceived delirium were twelve times more likely to have generalized anxiety symptomatology, but no associations between caregiver-perceived delirium and caregiver MDD, PTSD, or panic disorder were found.

\section{Associations with caregiver burden}

There was a statistically significant association between caregiver-perceived delirium and caregiver burden $(\beta=5.05$ [standard error $\{\mathrm{SE}\}=1.26$ ]; $p<$ $0.0001)$ with caregivers who caregiver-perceived delirium in the patient having higher burden on the CBS. On bivariate analysis, caregiver burden was strongly associated with generalized anxiety (odds ratio $[\mathrm{OR}]=1.13$ [confidence interval $\{\mathrm{CI}\}: 1.04,1.23$ ]; $p=0.006)$.

Table 2. Bivariate Regression Analyses for Caregiver-Perceived Delirium in Patients and Psychiatric Disorders in Caregivers $(N=200)$

\begin{tabular}{lllc}
\hline Outcome & $\%(\mathrm{n})$ & Odds ratio $(95 \%$ CI $)$ & p value \\
\hline Major depressive disorder & $7.0(14)$ & OR $=1.79(0.53-6.04)$ & 0.35 \\
Generalized anxiety & $3.5(7)$ & OR $=12.12(2.26-65.18)$ & 0.004 \\
Panic disorder & $7.5(15)$ & OR $=2.30(0.74-7.18)$ & 0.15 \\
Posttraumatic Stress Disorder & $6.0(12)$ & OR $=0.84(0.18-4.02)$ & 0.83 \\
\hline
\end{tabular}

${ }^{\mathrm{a}} \mathrm{GAD}$ symptomatology was only reported for 1 month, so subjects may not have had symptoms for 6 months that is required to make a formal diagnosis of GAD.

CI, confidence interval. 
Table 3. Caregiver-Perceived Delirium and Caregiver

Anxiety Adjusted for Caregiver Burden $(N=200)$

\begin{tabular}{llr}
\hline Caregiver anxiety & Odds ratio $(95 \%$ CI $)$ & p value \\
\hline Caregiver-perceived delirium & OR $=7.63(1.29-45.07)$ & 0.025 \\
Caregiver burden & OR $=1.09(0.99-1.20)$ & 0.069 \\
\hline
\end{tabular}

CI, confidence interval.

\section{Associations with generalized anxiety}

Of the 200 caregivers in the analysis, $(3.5 \%)$ met the SCID criteria for generalized anxiety. We found no association between GA and a variety of caregiver demographic variables, including age, gender, race, educational level, and health insurance status; therefore, we did not include these caregiver demographic variables in our regression models. Caregiver anxiety was not associated with patient Zubrod performance status, so we did not adjust for it in the subsequent analyses.

To characterize further the relationship between caregiver anxiety and caregiver-perceived delirium in patients, we added caregiver burden to the model. Caregiver-perceived delirium remained independently associated with caregiver anxiety, even after adjusting for caregiver burden, but the effect of caregiver burden on caregiver anxiety faded from a highly significant one to one of marginal significance $(p=0.07$; Table 3).

We conducted post hoc analyses to test for associations between caregiver anxiety and other symptoms on the SCARED. We found no significant associations between anxiety in caregivers and their report of observing the patient in pain, choking, dehydration, falls, or thinking the patient was dead. On bivariate analyses, vomiting, insomnia and thinking the patient had had enough were associated with increased caregiver anxiety (Table 4). In the final multivariate model estimating the relationship between caregiver-perceived delirium and caregiver anxiety, we included all the items on the SCARED and caregiver burden. The results (Table 4) demonstrate that only caregiver-perceived delirium remains associated with anxiety in caregivers.

\section{DISCUSSION}

This is the first study that systematically tests for an association between caregivers' perception of delirium in patients and rates of mental health problems in family caregivers of patients with advanced cancer. We found that caregivers who reported caregiver-perceived delirium in the patient were more likely to meet criteria for generalized anxiety. While caregiver reports of vomiting, insomnia, and feeling the patient had had enough were also associated with caregiver anxiety on univariate analyses, caregiver-perceived delirium was strongest predictor and the only one to remain associated on multivariate analyses. As expected, we also found a strong association between caregiver-perceived delirium and caregiver burden. The relationship between caregiver-perceived delirium and caregiver anxiety persists, even after adjusting for caregiver burden, indicating that the increased risk of

Table 4. Associations with Caregiver AnXiety

\begin{tabular}{|c|c|c|c|c|}
\hline \multirow[b]{2}{*}{ Items from SCARED ${ }^{\mathrm{a}}$} & \multicolumn{2}{|c|}{ Univariate analyses } & \multicolumn{2}{|c|}{ Multivariate analyses } \\
\hline & $\mathrm{p}$ value & Odds ratio $(\mathrm{CI})$ & $\mathrm{p}$ value & Odds ratio $(C I)$ \\
\hline Confused/delirious & 0.004 & $12.12(2.26-65.18)$ & 0.04 & $9.99(1.07-93.30)$ \\
\hline Experience severe pain/discomfort & 0.17 & $3.24(0.61-17.13)$ & 0.70 & $1.47(0.21-10.62)$ \\
\hline Unable to eat or swallow/Choking & 0.19 & $2.78(0.60-12.92)$ & 0.97 & $1.03(0.14-7.50)$ \\
\hline Vomiting & 0.04 & $5.28(1.11-25.06)$ & 0.10 & $6.85(0.70-67.20)$ \\
\hline Dehydration & 0.66 & $1.62(0.19-14.22)$ & 0.14 & $0.10(0.005-2.14)$ \\
\hline Insomnia & 0.05 & $5.28(1.00-27.99)$ & 0.13 & $4.73(0.63-35.37)$ \\
\hline Falling, passing out, collapsing & 0.19 & $4.43(0.47-41.91)$ & 0.62 & $0.44(0.02-11.77)$ \\
\hline Feeling the patient had had enough & 0.02 & $6.67(1.42-31.24)$ & 0.74 & $1.43(0.18-11.25)$ \\
\hline Thought the patient was dead & 0.28 & $3.39(0.37-31.21)$ & 0.48 & $0.33(0.02-6.87)$ \\
\hline Caregiver burden ${ }^{\mathrm{b}}$ & 0.006 & $1.13(1.04,1.23)$ & 0.14 & $1.09(0.97-1.22)$ \\
\hline
\end{tabular}

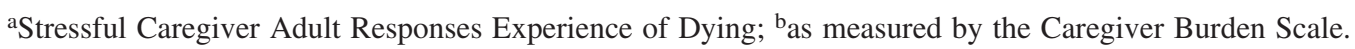


anxiety in caregivers of patients with caregiver-perceived delirium is not explained solely by the increased demands of care. The negative association of caregiver-perceived delirium with caregiver well-being has not been previously reported and has important implications.

\section{Clinical implications}

The association between caregiver-percieved delirium in patients and caregiver anxiety validates our clinical experience (M.K.B., S.D.B., S.K.I.) working with patients with advanced cancer and their family members. Delirium is a frightening event to experience or to witness, ${ }^{20,21}$ evoking fear and helplessness. This may explain why caregiver-perceived delirium was associated with anxiety and not depression, which would be more associated with feelings of sadness and loss. In mild cases of hypoactive delirium, the patient is withdrawn and "not himself." In more severe cases, the patient exhibits overt signs of apprehension, such as restlessness and verbal distress (e.g., screaming or moaning). In cases of hyperactive delirium, patients may become a danger to themselves (e.g., getting out of bed; pulling out intravenous lines), family, and staff (e.g., threatening or combative behavior) and unknowingly obstruct their own medical care. In all cases, delirium disrupts the preexisting relationship between the patient and the caregiver. In the setting of advanced cancer and a limited patient life expectancy, an abrupt disruption in this relationship may feel particularly threatening to caregivers as they anticipate the patient become sicker and fear future suffering for the patient. The caregiver may anticipate that the patient will never return to her/his baseline, or may not be able to effectively communicate again, raising the specter of permanent loss of the person, even before death. Additionally, the fluctuating nature of delirium means the patients may vacillate between being normal, or close to normal, and failing to recognize the caregiver. The inability of the caregiver to anticipate the state of the patient from hour to hour or day to day adds to the overall uncertainty of the patient's medical situation, and is likely to intensify caregiver stress and anxiety. The disruption of the patient-caregiver relationship and the uncertainty of restoring that relationship may explain why delirium, more than other common symptoms, such as pain, vomiting or insomnia, is associated with generalized anxiety in caregivers.

While the SCID was initially intended to make a diagnosis of GAD, for the purposes of this study, subjects were determined to have generalized anxiety based on symptoms they reported for the previous month. As a result, this study does not provide a diagnosis of GAD, nor does it distinguish generalized anxiety from other anxiety disorders, such as anxiety disorder not otherwise specified (NOS) and adjustment disorder with anxiety traits. Because GAD is considered a life-long disorder that typically surfaces in early adulthood $(D S M-I V)$, it would likely predate the presence of caregiver-perceived delirium. In contrast, the stressful experience of being a caregiver is thought to precipitate anxiety disorder (NOS) and adjustment disorder with anxiety trait in caregivers. Thus, in caregivers with a true diagnosis of GAD, the GAD would predate the report of patient symptoms and such a finding would imply that caregivers with GAD might overreport delirium symptoms. However, a diagnosis of another anxiety disorder leaves open the possibility that caregiver-perceived delirium in patients might precipitate anxiety in caregivers. This corroborates the findings in other studies where caregivers who observe delirium in patients describe the experience as a source of intense distress, guilt, and powerlessness. ${ }^{20,33}$

Knowing that caregiver-perceived delirium is associated with caregiver anxiety provides another reason to improve the diagnosis and management of delirium, which is often unrecognized and undertreated. ${ }^{34-36}$ As has been previously demonstrated regular nursing assessment of hospitalized oncology patients using a standardized screening tool to detect delirium can be done without undue burden on patients and allows earlier treatment of delirium, which may reduce the severity and the persistence of the patient's agitation and improve patient symptoms control. ${ }^{37,38}$ Additionally, a psycho-educational intervention among family caregivers of patients with delirium was well received by caregivers who overwhelmingly felt that the intervention should be offered to all caregivers of patients with delirium. ${ }^{33}$ Caregivers who received this intervention also reported improvement in medical decision-making. This kind of caregiver educational intervention, which informs caregivers about the nature of delirium, its fluctuating course and the hope of recovery, should be replicated. Such interventions could be expanded in a variety of ways, such offering emotional support during an episode of delirium. Families of patients with cancer could be encouraged to be present with their loved ones to help prevent delirium or minimize the agitation that occurs during an episode, ${ }^{39}$ however, family members need to be reassured that their presence is likely to be beneficial, even if the patient does not seem to acknowledge or appreciate their presence. Medical oncologists and nursing staff can provide specific instructions to family members about what to do and what not to do if the patient experiences delirium, 
making family caregivers feel less helpless and providing them with a greater sense of control of a frightening situation. Because delirium increases the burdens of caring for the patient, arrangements can be made to assist the caregiver with some of the physical burdens of the patient's care. Last, the disruption in a patient's decision-making capacity caused by delirium may serve as a trigger to discuss advance directives, identify a health care proxy and establish the role of further chemotherapy. Because delirium is a marker of poor prognosis in cancer patients, an episode of delirium may prompt oncologists to address and offer guidance about care wishes for the future. Further studies will need to be conducted to see if such interventions help alleviate caregiver anxiety.

\section{Research implications}

In addition to confirming clinical impressions, this study extends the current literature and suggests directions for further investigation. Our study is the first to describe a connection between caregiver-perceived delirium in patients and a mental health problem in caregivers. The relationship between caregiver-perceived delirium and caregiver psychological well-being should be further evaluated. Future studies should utilize validated measures of delirium, rather than relying only on reports of caregivers, and should also utilize a well-validated outcome measure to evaluate the impact of delirium on caregivers.

Additionally, our study extends the literature on the mental health of caregivers by finding that caregivers of delirious patients may be at increased risk for an anxiety disorder, rather then a depressive disorder. Previous literature examining the effects of caregiving, largely in populations of dementia caregivers, has focused on caregiver burden and depression as the primary outcomes of interest. ${ }^{10,40}$ Many studies of caregivers of cancer patients with advanced disease do not assess anxiety in caregivers. ${ }^{22}$ Future studies, examining caregiver wellbeing, should include measures of anxiety.

\section{Study limitations}

Our study design has several strengths and weaknesses. First, our study relies on retrospective recollection of caregivers about seeing a patient being confused or delirious as a measure of delirium. Unfortunately, information on past medical history of delirium, which is known to correlate with subsequent delirium, was not available from our dataset. The ability of our caregiver-perceived delirium measure to distinguish between delirium and dementia is unclear. The correlation of caregiver report of delirium and delirium diagnosed by a clinician is unknown, but caregivers have been shown to be accurate at assessing cognitive impairment in patients. ${ }^{41}$ Although caregivers may not be able to distinguish between delirium and dementia, the study entry criteria were designed to exclude patients with dementia from enrollment in the study. Study exclusion criteria, an adequate score on the SPMSQ and the rigor of the interview all argue against the presence of significant dementia in these patients. Additionally, the caregiver's report of delirium was associated with a lower SPMSQ score, and worse performance status, which have been shown to correlate with delirium ${ }^{27,42}$ and lend validity to our measure, of caregiver-perceived delirium. When we found the strong statistical relationship between anxiety and caregiver-perceived delirium, we looked for support of the alternate hypothesis that caregivers with generalized anxiety might be prone to over-report confusion or delirium in patients. We postulated that the overreporting bias would not be unique to reported delirium but would occur in response to other caregiver exposures as well. However, when we examined this question, we found that caregivers with generalized anxiety were not more likely to report witnessing other forms of patient distress, such as pain, vomiting or falls on a multivariate analysis. As a cross-sectional study, we cannot fully address the issue of causation, but our findings do not support the hypothesis that anxiety leads to an overreporting bias of other types of stressful experiences in terminally ill patients. A planned analysis of longitudinal data, when it becomes available, will determine more conclusively the direction of causality.

While we had a large sample of cancer caregivers on which to conduct our study $(n=200)$, those with reported symptoms of delirium $(n=38)$ and those with generalized anxiety $(n=7)$ were relatively few in number. The small number of subjects with the primary outcome of interest (generalized anxiety) limits our ability to conduct analyses and puts us at risk for overtesting. Additionally, even if we were able to prove (which our cross-sectional data cannot) causality from caregiver-perceived delirium to caregiver anxiety, we recognize that caregiver-perceived delirium would be only one of many factors that could contribute to caregiver anxiety. For example, caregivers who are already predisposed to mental health problems may be more susceptible to developing generalized anxiety, after being exposed to delirium in a patient. However, the persistence of the relationship between caregiver-perceived delirium in the patient and caregiver anxiety in bivariate and two multivariate models lend validity to our findings. 


\section{CONCLUSION}

Our findings of an association between caregiverperceived delirium in patients and generalized anxiety in caregivers support the hypothesis that the development of delirium leads to increased anxiety in caregivers. This is the first report of an association between reports of patient delirium and a caregiver mental health outcome. Further studies using a validated tool to measure delirium and longitudinal data collection are warranted.

\section{ACKNOWLEDGMENTS}

This research was supported in part by the following grants: MH56529 (H.G.P.) and MH63892 (H.G.P.) from the National Institute of Mental Health and CA106370 (H.G.P.) from the National Cancer Institute; K24AG00949 (S.K.I.) from the National Institute on Aging; the Center for Psycho-oncology and Palliative Care Research, Dana-Farber Cancer Institute (H.G.P., L.C.V., B.Z., M.K.B.). Dr. Inouye is supported by the Milton and Shirley F. Levy Family Chair.

\section{REFERENCES}

1. Bruera E, Miller L, McCallion J, Macmillan K, Krefting $\mathrm{L}$, Hanson $\mathrm{J}$ : Cognitive failure in patients with terminal cancer: A prospective study. J Pain Symptom Manage 1992;7:192-195.

2. Fann JR, Alfano CM, Burington BE, Roth-Roemer S, Katon WJ, Syrjala KL: Clinical presentation of delirium in patients undergoing hematopoietic stem cell transplantation. Cancer 2005; 103:810-820.

3. Fann JR, Roth-Roemer S, Burington BE, Katon WJ, Syrjala KL: Delirium in patients undergoing hematopoietic stem cell transplantation. Cancer 2002;95:1971-1981.

4. Pereira J, Hanson J, Bruera E: The frequency and clinical course of cognitive impairment in patients with terminal cancer. Cancer 1997;79:835-842.

5. Lawlor PG, Gagnon B, Mancini IL, Pereira JL, Hanson J, Suarez-Almazor ME, Bruera ED: Occurrence, causes, and outcome of delirium in patients with advanced cancer: a prospective study. Arch Intern Med 2000;160:786-794.

6. Caraceni A, Nanni O, Maltoni M, Piva L, Indelli M, Arnoldi E, Monti M, Montanari L, Amadori D, De Conno F: Impact of delirium on the short term prognosis of advanced cancer patients. Italian Multicenter Study Group on Palliative Care. Cancer 2000;89:1145-1149.

7. Morita T, Tsunoda J, Inoue S, Chihara S: Survival prediction of terminally ill cancer patients by clinical symptoms: Development of a simple indicator. Jpn J Clin Oncol 1999;29:156-159.

8. Marcantonio ER, Goldman L, Mangione CM, Ludwig LE,
Muraca B, Haslauer CM, Donaldson MC, Whittemore AD, Sugarbaker DJ, Poss R, et al: A clinical prediction rule for delirium after elective noncardiac surgery. JAMA 1994; 271:134-139.

9. Schulz R, Newsom J, Mittelmark M, Burton L, Hirsch C, Jackson S: Health effects of caregiving: the caregiver health effects study: An ancillary study of the Cardiovascular Health Study. Ann Behav Med 1997;19:110-116.

10. Schulz R, O'Brien AT, Bookwala J, Fleissner K: Psychiatric and physical morbidity effects of dementia caregiving: Prevalence, correlates, and causes. Gerontologist 1995; 35:771-791.

11. Shigenobu K, Ikeda M, Fukuhara R, Maki N, Hokoishi K, Nebu A, Komori K, Tanabe H: Reducing the burden of caring for Alzheimer's disease through the amelioration of "delusions of theft" by drug therapy. Int J Geriatr Psychiatry 2002;17:211-217.

12. Rabins PV, Fitting MD, Eastham J, Fetting J: The emotional impact of caring for the chronically ill. Psychosomatics 1990;31:331-336.

13. Schulz R, Mendelsohn AB, Haley WE, Mahoney D, Allen RS, Zhang S, Thompson L, Belle SH: End-of-life care and the effects of bereavement on family caregivers of persons with dementia. N Engl J Med 2003;349:1936-1942.

14. Coen RF, Swanwick GR, O’Boyle CA, Coakley D: Behaviour disturbance and other predictors of carer burden in Alzheimer's disease. Int J Geriatr Psychiatry 1997;12:331336.

15. Shua-Haim JR, Haim T, Shi Y, Kuo YH, Smith JM: Depression among Alzheimer's caregivers: Identifying risk factors. Am J Alzheimers Dis Other Demen 2001;16:353359.

16. Sansoni J, Vellone E, Piras G: Anxiety and depression in community-dwelling, Italian Alzheimer's disease caregivers. Int J Nurs Pract 2004;10:93-100.

17. Coyne AC, Reichman WE, Berbig LJ: The relationship between dementia and elder abuse. Am J Psychiatry 1993; 150:643-646.

18. Zarit SH, Todd PA, Zarit JM: Subjective burden of husbands and wives as caregivers: a longitudinal study. Gerontologist 1986;26:260-266.

19. Rankin ED, Haut MW, Keefover RW, Franzen MD: The establishment of clinical cutoffs in measuring caregiver burden in dementia. Gerontologist 1994;34:828-832.

20. Breitbart W, Gibson C, Tremblay A: The delirium experience: Delirium recall and delirium-related distress in hospitalized patients with cancer, their spouses/caregivers, and their nurses. Psychosomatics 2002;43:183-194.

21. Morita T, Hirai K, Sakaguchi Y, Tsuneto S, Shima Y: Family-perceived distress from delirium-related symptoms of terminally ill cancer patients. Psychosomatics 2004;45: 107-113.

22. Vanderwerker LC, Laff RE, Kadan-Lottick NS, McColl S, Prigerson HG: Psychiatric disorders and mental health service use among caregivers of advanced cancer patients. J Clin Oncol 2005;23:6899-6907.

23. Charlson M, Szatrowski TP, Peterson J, Gold J: Validation of a combined comorbidity index. J Clin Epidemiol 1994;47:1245-1251.

24. Prigerson HG, Cherlin E, Chen JH, Kasl SV, Hurzeler R, 
Bradley EH: The Stressful Caregiving Adult Reactions to Experiences of Dying (SCARED) Scale: A measure for assessing caregiver exposure to distress in terminal care. Am J Geriatr Psychiatry 2003;11:309-319.

25. Pfeiffer E: A short portable mental status questionnaire for the assessment of organic brain deficit in elderly patients. J Am Geriatr Soc 1975;23:433-441.

26. Fillenbaum GG: Comparison of two brief tests of organic brain impairment, the MSQ and the short portable MSQ. J Am Geriatr Soc 1980;28:381-384.

27. Fillenbaum GG, Landerman LR, Simonsick EM: Equivalence of two screens of cognitive functioning: The Short Portable Mental Status Questionnaire and the OrientationMemory-Concentration test. J Am Geriatr Soc 1998;46: 1512-1518.

28. Erkinjuntti T, Sulkava R, Wikstrom J, Autio L: Short Portable Mental Status Questionnaire as a screening test for dementia and delirium among the elderly. J Am Geriatr Soc 1987;35:412-416.

29. Williams JB, Gibbon M, First MB, Spitzer RL, Davies M, Borus J, Howes MJ, Kane J, Pope HG Jr, Rounsaville B, et al: The Structured Clinical Interview for DSM-III-R (SCID). II. Multisite test-retest reliability. Arch Gen Psychiatry 1992;49:630-636.

30. Andren S, Elmstahl S: Family caregivers' subjective experiences of satisfaction in dementia care: Aspects of burden, subjective health and sense of coherence. Scand J Caring Sci 2005;19:157-168.

31. Elmstahl S, Malmberg B, Annerstedt L: Caregiver's burden of patients 3 years after stroke assessed by a novel caregiver burden scale. Arch Phys Med Rehabil 1996;77:177182.

32. Lawlor PG, Gagnon B, Mancini IL, Pereira JL, Hanson J, Suarez-Alamazor M, Bruera ED: Delirium as a predictor of survival in older patients with advanced cancer. Arch Intern Med 2000;160:2866-2868.

33. Gagnon P, Charbonneau C, Allard P, Soulard C, Dumont S, Fillion L: Delirium in advanced cancer: A psychoeducational intervention for family caregivers. J Palliat Care 2002;18:253-261.

34. Inouye SK: Delirium in older persons. $\mathrm{N}$ Engl J Med 2006;354:1157-1165.
35. Inouye SK, Foreman MD, Mion LC, Katz KH, Cooney LM Jr: Nurses' recognition of delirium and its symptoms: Comparison of nurse and researcher ratings. Arch Intern Med 2001;161:2467-2473.

36. Margolis RL: Nonpsychiatrist house staff frequently misdiagnose psychiatric disorders in general hospital inpatients. Psychosomatics 1994;35:485-491.

37. Gaudreau JD, Gagnon P, Harel F, Tremblay A, Roy MA: Fast, systematic, and continuous delirium assessment in hospitalized patients: The nursing delirium screening scale. J Pain Symptom Manage 2005;29:368-375.

38. Gagnon P, Allard P, Masse B, DeSerres M: Delirium in terminal cancer: A prospective study using daily screening, early diagnosis, and continuous monitoring. J Pain Symptom Manage 2000;19:412-426.

39. Caplan GA, Coconis J, Board N, Sayers A, Woods J: Does home treatment affect delirium? A randomised controlled trial of rehabilitation of elderly and care at home or usual treatment (The REACH-OUT trial). Age Ageing 2006;35: 53-60.

40. Schulz R, Martire LM: Family caregiving of persons with dementia: Prevalence, health effects, and support strategies. Am J Geriatr Psychiatry 2004;12:240-249.

41. Fischer L, Visintainer PF, Schulz R: Reliable assessment of cognitive impairment in dementia patients by family caregivers. Gerontologist 1989;29:333-335.

42. Metitieri T, Bianchetti A, Trabucchi M: Delirium as a predictor of survival in older patients with advanced cancer. Arch Intern Med 2000;160:2866-2868.

Address reprint requests to: Mary K. Buss, M.D. Dana-Farber Cancer Institute Thoracic Oncology Program Division of Psychosocial Oncology and Palliative Care 44 Binney Street, SW 411 Boston, MA 02115

E-mail: mary_buss@dfci.harvard.edu 
Copyright of Journal of Palliative Medicine is the property of Mary Ann Liebert. Inc. and its content may not be copied or emailed to multiple sites or posted to a listserv without the copyright holder's express written permission. However, users may print, download, or email articles for individual use. 